\title{
Unilateral Absence of Ovary and Fallopian Tube: 3 Rare Case Reports
}

\author{
MARUF SIDDIQUI ${ }^{1}$, NUSRAT GHAFOOR ${ }^{2}$, FAHMIDANAZNINE ${ }^{3}$, \\ CHOWDHURY AYESHA SIDDIQUA ${ }^{4}$, TA CHOWDHURY ${ }^{5}$
}

\begin{abstract}
:
Here we reported three cases of unilateral tubal and ovarian absence, along with laparoscopic images and images during ceasarean section in Anwer Khan Modern Medical College. Three patients with primary infertility of 6-8 years duration were reported as non-visualization of right adnexal structures on transvaginal ultrasound and hysterosalpingography. Absence of right adnexal structures of varying degree were detected during laparoscopy. Subsequent evaluation of renal system with Intravenous Pyelography were done. Ipsilateral absence of the fallopian tube and ovary without any other system anomalies were detected. Vascular accidents, Congenital defects or torsion could be the possible etiologic factors. Laparoscopy is a feasible option as a diagnostic tool for these cases.
\end{abstract}

Key Words: Unilateral Absence, Fallopian tube, Ovary, Congenital anomaly

\section{Introduction:}

Unilateral absence of ovary and fallopian tube is an extremely rare occurrence with only a very few cases reported in literatures. Transvaginal sonography may demonstrate non visualization of adnexal structures and arise suspicion about this type of anomaly. But, the only way to diagnose and confirm this type of anomaly is usually diagnostic laparoscopy done on an infertile patient. Some authors have suggested that the incidence is about 1 in $11,240^{1,2}$. The incidence may be much higher considering the fact that many cases remain undiagnosed. This article reports three cases of unilateral absence of ovary and fallopian tubes that were diagnosed by laparoscopy performed on infertile patients. Possible causes are discussed and documented.

\section{Case Reports}

\section{Case 1}

A 26 years old patient presented to the Department of Infertility and Reproductive Medicine, Anwer Khan Modern Medical College with history of 5 years of primary subfertility. On pelvic examination, cervix and vagina were normal, uterus was of normal size and mobile, bilateral fornices were free and non tender. Patient did not have any history of abdominal or pelvic surgery.

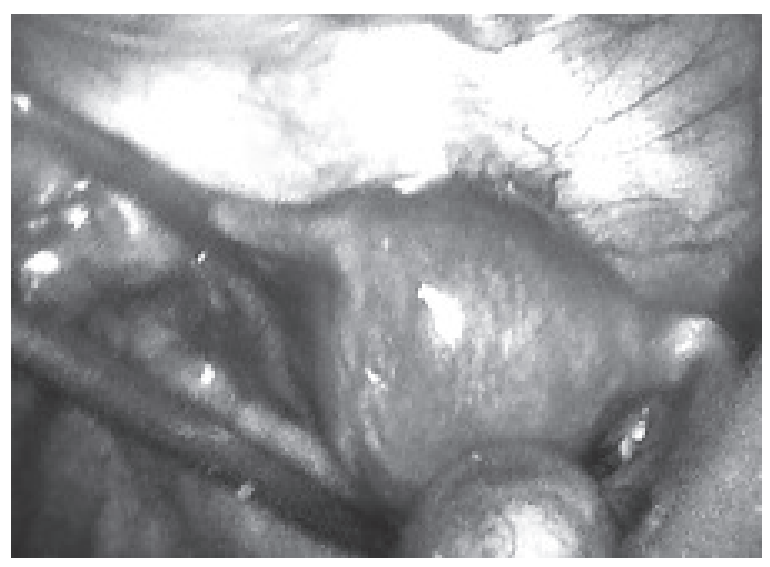

Fig-1: Laparoscopoc view of $1^{\text {st }}$ case showing absent right ovary and tube.

1. Associate Professor \& Head, Department of Infertility \& Reproductive Medicine, Anwer Khan Modern Medical College

2. Associate Consultant \& Assistant Professor, Department of Radiology \& Imaging, Ibrahim Cardiac Hospital \& Research Institute(ICHRI)

3. Assistant Registrar, Department of Infertility \& Reproductive Medicine, Anwer Khan Modern Medical College

4. Medical Officer, Department of Infertility \& Reproductive Medicine, Anwer Khan Modern Medical College

5. Chief Consultant \& Professor of Obstetrics \& Gynaecology, Ibrahim Medical College \& BIRDEM Hospital 
Day 21 progesterone confirmed that this patient was ovulating regularly. Hysterosalpingography demonstrated non-visualization of the right fallopian tube and no spillage was observed from the left side (Cornual Block). Transvaginal sonography could not detect the right ovary. For reevaluation of tubes laparoscopy was decided. On laparoscopy, uterus was normal, left sided tube and ovary were normal, but on the right side, both tube and ovary were absent (Fig.-1). Dye test was done. There was obviously no spillage from the right side. But though initially negative, following pressure and repeated trial, dye passed freely from the left tube with expulsion of large amounts of mucous plug. There was no adhesion surrounding it. Round ligament, uterosacral ligament and pouch of Douglas were normal. Intravenous pyelogram was done and no renal abnormalities were detected. Following laparoscopy, ovulation induction was given with follicular monitoring and timed intercourse. She conceived on sixth cycle of ovulation induction coupled with intrauterine insemination (IUI). Caesarean section during delivery also confirmed the same findings of laparoscopy.

\section{Case 2}

A 29 years old patient presented to the Department of Infertility and Reproductive Medicine, Anwer Khan Modern Medical College with history of 6 years of primary subfertility. On pelvic examination, cervix and vagina were normal, uterus was of normal size and mobile, bilateral fornices were free and non tender. Patient did not have any history of abdominal or pelvic surgery.

Day 21 progesterone of this patient indicated anovulation. Hysterosalpingography demonstrated non-visualization of the right fallopian tube and a patent left tube. Transvaginal sonography could not detect the right ovary but demonstrated an enlarged and polycystic left ovary. After 6 cycles of ovulation induction laparoscopy was decided. On laparoscopy, uterus was normal, left sided tube was normal, left ovary was polycystic and on the right side, both tube and ovary were absent. Dye test was done. There was obviously no spillage from the right side but dye passed freely from the left tube. A small fold of loose tissue like bulging was found on the right ovarian fossa. It was dissected and no structure was found. There were no adhesion surrounding it. Round ligament, uterosacral ligament and pouch of Douglas were normal (Fig.-2). Intravenous pyelogram was done and no renal abnormalities were detected. Following laparoscopy, ovulation induction was given with Letrozole and recombinant Gonadotropins. After follicular monitoring patient was advised for timed intercourse. This patient also conceived on 4th cycle of ovulation induction. Caesarean section was done and during delivery the same findings of laparoscopy was confirmed (Fig.-3).

\section{Case 3}

A 28 years old patient presented to the Department of Infertility and Reproductive Medicine, Anwer Khan Modern Medical College with history of 4 years of

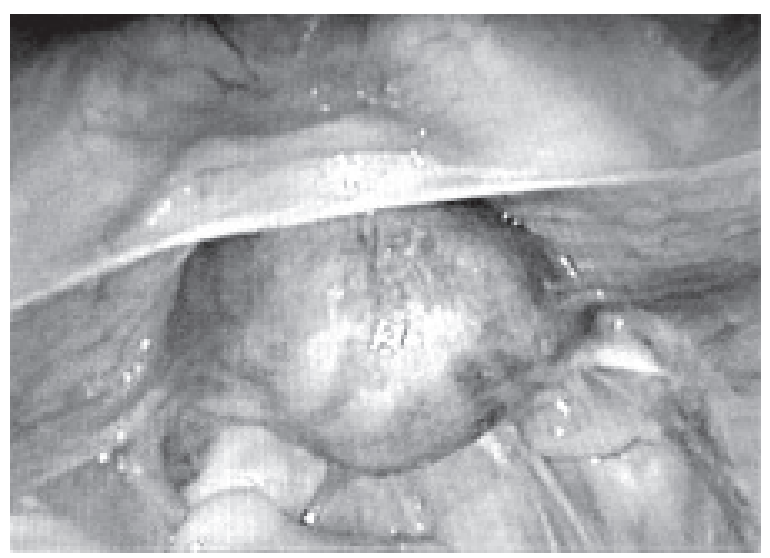

Fig.-2: Laparoscopic view of $2^{\text {nd }}$ case showing a polycystic left ovary and small fold of tissue in the Right ovarian fossa

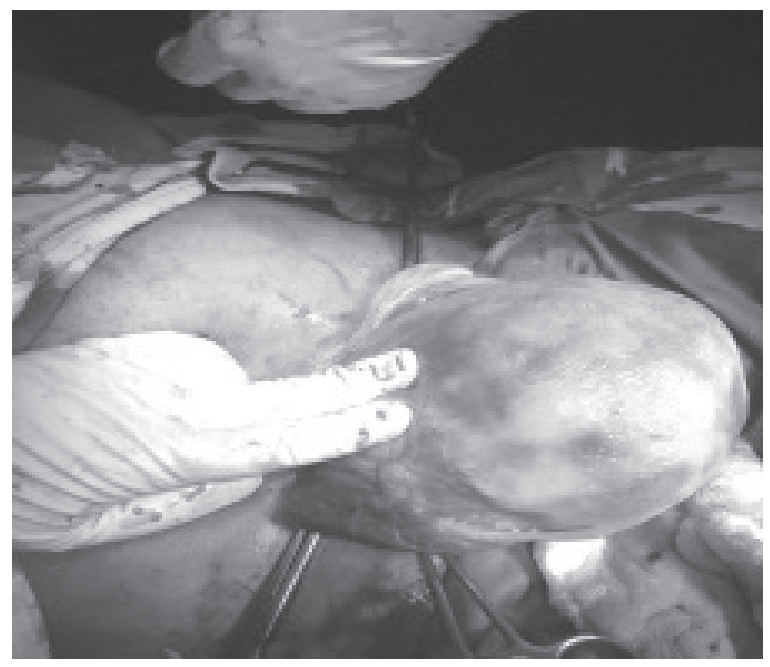

Fig.-3: Photograph taken during caesarean section in the $2^{\text {nd }}$ case again showing the same anomaly 
primary subfertility. On pelvic examination, cervix and vagina were normal, uterus was of normal size and mobile, bilateral fornices were free and non tender. Patient did not have any history of abdominal or pelvic surgery.

Day 21 progesterone of this patient indicated regular ovulations. Hysterosalpingography demonstrated nonvisualization of both tubes and no spillage on any of the side. Transvaginal sonography could not detect the right ovary but demonstrated a normal left ovary . For re-evaluation of tubes laparoscopy was decided. On laparoscopy, uterus was normal, right tube and ovary were absent. Left ovary was of normal size with only a rudimentary part of the left tube. There were no adhesions surrounding it. Round ligament, uterosacral ligament and pouch of Douglas were normal (Fig.-4). Following laparoscopy, Intravenous pyelogram was done and no renal abnormalities were detected. At present, this patient is awaiting to undergo In vitro fertilization (IVF).

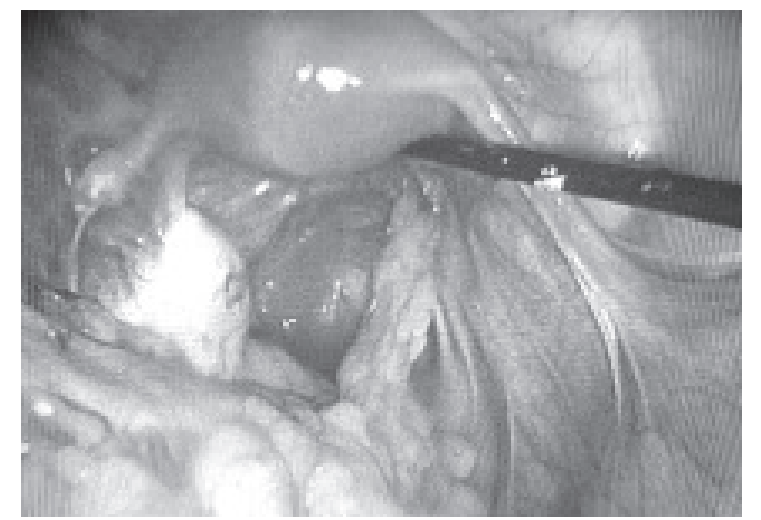

Fig.-4: Laparoscopic view of Case 3 showing absence of Right ovary \& tube. The left ovary is normal with a residual portion of left tube is also seen.

\section{Discussion:}

The exact etiology of ipsilateral tubal and ovarian absence remains a mystery. Reported cases to date have suggested three possible etiologies: a) Adnexal torsion, b). Tubal and ovarian maldevelopment secondary to ischaemia due to a vascular accident, and 3) A defect in the development of the mullerian and mesonephric system, either entirely or on one side or localized to the region of genital ridge and the caudal part. ${ }^{1,3-5}$

Adnexal torsion is usually associated with sever abdominal pain during childhood, in adults and can be seen also during pregnancy. However, Sivanesaratnam ${ }^{1}$ suggested that adnexal torsion might occur during fetal life. In the present case there was no history of acute abdominal pain; however, the absence of symptoms does not exclude the possibility of a torsion that had occurred during fetal life.

Few cases have been reported in the literature with various clinical features. Uckuyu et al. ${ }^{3}$ reported that their cases also did not have a history of acute pelvic pain, but they pointed out that the torsion hypothesis could also be supported by the finding of separated structures in the abdominal cavity, such as tubal and ovarian tissue remnants. They have suggested that torsion might be the possible etiologic factor owing to the presence of partial agenesis or a twisted-elongated tube, whereas a normal tube was present on the contralateral side in all of their cases. However, bilateral normal ovaries were detected during laparoscopy in one of their cases, so the torsion hypothesis was debatable. On the other hand, Mylonas et al. ${ }^{4}$ proposed that vascular accident or failed canalization of the upper part of one fallopian tube might also be responsible for the agenesis of ipsilateral fallopian tube and ovary.

Cases with absent fallopian tube and/or ovary that were associated with either urinary or gastrointestinal system anomalies have been reported ${ }^{5-8}$. Additionally, uterine malformations with or without round ligament anomalies have also been reported within the scope of these cases $^{5,7,9,10}$.In our three cases, we could not find any anomaly in the uterus or in the renal system. However, similar to ours, cases that were not associated with any other system anomalies have been reported $3,4,9$. However, the absence of any other system anomalies and absence of torsion-related past acute abdominal pain makes us think that vascular accident stands in the forefront of suggested etiologies.

Our case 1 conceived, though she had unilateral absence of ovary and fallopian tube, laparoscopy was able to open the left tube. She conceived following ovulation induction coupled with intrauterine insemination, which was done to increase the chances of pregnancy when a mature follicle on the left ovary was found on Day 12 folliculometry.

The basic cause of infertility in case 2 was anovulation due to a single polycystic ovary on the left side. Based on findings of ultrasonography and hysterosalpingography laparoscopic evaluation was done. As left tube was patient after successful induction of 
ovulation pregnancy occurred. Due to tubal block $3^{\text {rd }}$ case was selected for IVF and ET.

\section{Conclusion:}

Ipsilateral absence of the fallopian tube and ovary should be kept in mind if the adnexa of one side cannot be visualized by imaging techniques. This rare condition may be associated with infertility, especially for patients with contralateral tubal pathologies. Finally, laparoscopy is a feasible diagnostic tool for these cases.

\section{References:}

1. Sivanesaratnam V. Unexplained unilateral absence of ovary and fallopian tube. Eur J Obstet Gynecol Reprod Med. 1986;22:103-105

2. Pabuccu E $E^{1}$, Kahraman K, Task1n S, Atabekoglu C.Unilateral absence of fallopian tube and ovary in an infertile patient. Fertil Steril. 2011 Jul;96(1):e55-7

3. Uckuyu, A., Ozcimen, E.E., and Sevinc Ciftci, F.C. Unilateral congenital ovarian and partial tubal absence: report of four cases with review of the literature. Fertil Steril. 2009; 91: 936.e5-936.e8

4. Mylonas, I., Hansch, S., Markmann, S., Bolz, M., and Friese, K. Unilateral ovarian agenesis: report of three cases and review of the literature.
Arch Gynecol Obstet. 2003; 268: 57-60

5. Muppala, H., Sengupta, S., and Martin, J.E. Unilateral absence of tube and ovary with renal agenesis and associated pyloric stenosis: communication. Eur J Obstet Gynecol Reprod Biol. 2008; 137: 123

6. Zaitoon, M.M. and Florentin, H. Crossed renal ectopia with unilateral agenesis of fallopian tube and ovary. J Urol. 1982; 128

7. Haydardedeoglu, B., Simsek, E., K1l1cdag, E., Tar1m, E., Aslan, E., and Bagis, T. A case of unicornuate uterus with ipsilateral ovarian and renal agenesis. Fertil Steril. 2006; 85: 750.e1750.e4

8. Mulay1m, B., Demirbasoglu, S., and Oral, O. Unicornuate uterus and unilateral ovarian agenesis associated with pelvic kidney. Surg Endosc. 2003; 17: 161

9. Rapisarda, G., Pappalardo, E.M., Arancio, A., and La Greca, M. Unilateral ovarian and fallopian tube agenesis. Arch Gynecol Obstet. 2009; 280: 849-850

10. Suh B, Kalam M. Septate uterus with left fallopian tube hypoplasia and ipsilateral ovarian agenesis. J Assist Reprod Genet 2008;25: 567-9 